NMR spectroscopy played a crucial role in verifying the growth of the Zn-rich outer shell. First, ${ }^{13} \mathrm{C}$ MAS spectra with proton cross polarization with and without dipolar dephasing showed that the organic capping ligand, stearate, is firmly attached by the carboxyl group to the surface, with only the extremities of the alkyl chain showing any signs of motion. (This contrasts with earlier observations of trioctylphosphine oxide capping molecules hopping around on the surface of CdSe and CdTe QDs). With attached organic groups it was then possible to obtain ${ }^{113} \mathrm{Cd}$ MAS spectra with and without ${ }^{1} \mathrm{H}$ cross polarization and thus distinguish surface versus core $\mathrm{Cd}$ atoms. The signal of surface Cds with coordinated carboxyl groups was observed to decrease as the $\mathrm{Zn}$ content of the QD increased, and eventually disappeared when there was more $\mathrm{Zn}$ than $\mathrm{Cd}$. The disappearance of this signal attests to the depletion of $\mathrm{Cd}$ in the surface layers, since Cd should still be visible if it were present in the surface layers in the same ratio as the overall $\mathrm{Zn}$ :Cd composition. An increasing chemical shift of the signal of the core Cd also demonstrated a $\mathrm{ZnCdS}$ alloy with increasing $\mathrm{Zn}$ content.

According to the researchers, the study describes the synthesis-structureproperty relationships of ternary $\mathrm{ZnCdS}$ alloys. Ternary ZnCdS QDs, with intense PL emission and narrow full width at half maximum, are excellent candidates as blue luminescent materials for various applications, and this study presents a new and easy method for their synthesis, said the researchers.

GOPAL RAO

\section{CNT Turfs Demonstrate Coordinated Buckling}

Carbon nanotubes (CNTs) have had an impact on multidisciplinary scientific fields, and their properties have led to widespread use in structural, electrical, and thermal applications. Dense fields of intertwined, vertically-oriented carbon nanotubes are known as CNT turfs. Realizing the importance of studying the mechanical behavior of turfs bonded to a semiconductor-based substrate, A.A. Zbib, S.Dj. Mesarovic, and D.F. Bahr of Washington State University; E.T. Lilleodden of the GKSS Research Center, Germany; and D. McClain and J. Jiao of Portland State University have recently prepared patterns of vertically aligned CNTs on photolithographically prepared silicon wafers.

The mechanical properties of individual single-walled and multi-walled CNTs have been studied both experimentally and theoretically using simulations like molecular dynamics and finite element methods in tension, compression, or bending. However, the collective behavior of complex CNT geometries like CNT turfs has not received considerable attention. CNT turfs are complex structures of interwined and nominally vertical tubes. The behavior of freestanding CNT turfs has been reported, but their potential use in contact switches necessitates further study. In the April 30 issue of Nanotechnology (DOI: 10.1088/0957), the researchers described the coordinated buckling behavior of collective CNT segments using experimental and theoretical approaches.

In the experiments, CNT turfs were grown using chemical vapor deposition from a catalyst containing glass. This led to turfs that were bonded to the growth substrate. The researchers measured the mechanical properties of turfs using localized compression by nanoindentation and uniform compression. They measured the tangent elastic modulus of CNT turfs with a blunt Berkovich tip of a $1000 \mathrm{~nm}$ radius using a triboscope in conjunction with a scanning probe microscope. Although the elastic modulus of a single CNT can be on the order of $1 \mathrm{TPa}$, researchers reported an average tangent modulus of $\sim 15 \mathrm{MPa}$. This can be expected from a collective response of CNTs in a turf, which is dominated by buckling/ bending of CNT segments. Uniform compression experiments were done to place an entire column width in compression, and coordinated reorientation and collective buckling in the layer were observed. Various turfs with $300 \mu \mathrm{m}$ diameters and heights varying from $25 \mu \mathrm{m}$ to $204 \mu \mathrm{m}$ were tested using a displacementcontrolled compression tool. Other turf samples with $30 \mu \mathrm{m}$ turf radii and $\sim 60 \mu \mathrm{m}$ heights were compression-tested using load-controlled nanoindentation, employing a flat diamond punch.

The researchers developed a mechanical model that describes the buckling of these complex turfs, and the researchers were able to validate their findings from scanning electron microscope images. Researchers concluded that CNT turfs exhibit coordinated buckling in the bottom layer under uniform compression at nominal stresses of between $4.3 \mathrm{MPa}$ and $0.2 \mathrm{MPa}$.

"The buckling stress depends mainly on the effective tangent modulus of the turf, its height, and its nanogeometry," the researchers said. They said that their findings should help in materials selection and design of CNT-based materials that rely on the collective mechanical behavior of CNT turfs such as switches or future CNT integration into microelectronic packaging to take advantage of the thermal and electric conductivity of nanotubes.

ROHIT KHANNA

\section{Nanosized Optical Emitter Coupled to Gold Nanowires Enhances the Coupling Constant and the Surface Plasmon Density of States}

Coupling of quantum systems with photon fields is crucial to develop quantum information processing tasks. There has recently been significant interest in using surface plasmons (SP) to propagate information through systems of nanowires. SP emission scales with decreasing nanowire radius, but so do SP propagation losses. Consequently, very small wires have not proven suitable for long-distance transmission of information via SP. S.D. Liu, M.T. Cheng, Z.J. Yang, and Q.Q. Wang from the Wuhan University, China, have demonstrated theoretically that a pair of nanowires of relatively large radii show a stronger coupling with an emitter and present smaller losses for SP propagation than when using only a single nanowire.

As reported in the April 15 issue of Optics Letters (p. 851), the researchers used the finite difference time domain (FDTD) simulation method to study the SP coupling, SP propagation, and far field emission for an oscillating dipole emitter coupled to a single and a pair of gold nanowires, with the emitter located at a distance $\Delta$ from the surface of the nanowires, and forming an angle $\theta$ with their axes.

The researchers showed that when using a pair of nanowires, the coupling with the emitter is stronger and the losses of SP propagation can be reduced. They also showed that the field could not be enhanced when the polarization of the emitter was vertical to the connection line between the central point of the two nanowires $\left(\theta=0^{\circ}\right)$, while it was significantly excited when $\theta=90^{\circ}$. At the same time, the spontaneous emission rate became faster, and while the SP wavelength $\left(\lambda_{\mathrm{SP}}\right)$ was found to be constant for a single wire, the simulation results revealed that $\lambda_{\mathrm{SP}}$ could be tuned from $650 \mathrm{~nm}$ to $380 \mathrm{~nm}$ when the distance between the two nanowires was reduced from $60 \mathrm{~nm}$ to $5 \mathrm{~nm}$. The generated plasmon field, which was proportional to the total spontaneous emission rate when coupling with one nanowire, increased for a pair of nanowires. The researchers then showed that the converted SP energy and the far field emission intensity of a pair of gold nanowires increased by about 4 times compared to those of a single nanowire.

These calculations demonstrated that the tight confinement between the two gold nanowires enhanced the coupling constant and the SP density of states, resulting in a stronger coupling without reducing their radii, and allowing SP to be transmitted over long distances.

JOAN J. CARVAJAL 